\title{
Angioplastia Primária via Radial com Doses Aumentadas de Tirofiban: uma Técnica Segura e Efetiva
}

\author{
Marden Andre Tebet ${ }^{1,2}$, Pedro Beraldo de Andrade ${ }^{1,2}$, Milena Gentile ${ }^{1}$, \\ Luiz Alberto Mattos ${ }^{1,3}$, André Labrunie ${ }^{1,2}$
}

\section{RESUMO}

Introdução: O acesso transradial para a realização de angioplastia primária é eficaz e seguro, mesmo com utilização de um regime agressivo de anticoagulação e antiagregação. O objetivo dessa série histórica de casos é avaliar a segurança e a efetividade da angioplastia primária via radial associada à utilização de bolus com dose aumentada de tirofiban. Método: Foram avaliados, retrospectivamente, no período de janeiro de 2006 a abril de 2007, 73 pacientes submetidos à angioplastia primária, em nosso serviço, pelo acesso radial, com análise dos resultados hospitalares e tardios, com enfoque nas complicações hemorrágicas e vasculares. Resultados: Obteve-se sucesso de $100 \%$ na utilização da via de acesso radial. Sucesso angiográfico ocorreu em $95,8 \%$ dos pacientes. A artéria coronária direita foi tratada em $50 \%$ dos casos. Tirofiban com bolus aumentado foi utilizado em $75 \%$ dos pacientes. Durante a fase hospitalar, houve três óbitos de causa cardíaca e nenhum caso de sangramento maior. Hematomas localizados ocorreram em $5,4 \%$ dos casos. No seguimento tardio (média de 7 meses), a taxa de mortalidade foi de $5,4 \%$, sem nenhuma complicação relacionada ao sítio de punção arterial ou sangramento maior. Conclusão: No nosso serviço de cardiologia intervencionista, que utiliza a via radial sistematicamente, seu emprego na angioplastia primária é altamente eficaz e seguro, a despeito do uso de um regime de anticoagulação e antiagregação agressivos.

DESCRITORES: Angioplastia transluminal percutânea coronária. Artéria radial. Tirosina, análogos \& derivados.

- Serviço de Hemodinâmica da Santa Casa de Marília, Marília, SP.

2 Hospital do Coração de Londrina, Londrina, PR.

3 Instituto Dante Pazzanese de Cardiologia, São Paulo, SP.

Correspondência: Marden André Tebet. Avenida Carlos Artêncio, 356 - Apto. 43B - Marília, SP - CEP 17519-255

E-mail: mardentebet@uol.com.br

Recebido em: 10/6/2007 • Aceito em: 27/7/2007
SUMMARY

Primary Angioplasty Using the Radial Access With High Bolus Dose of Tirofiban: a Safe and Effective Technique

Background: The use of the transradial access for primary angioplasty is efficient and safe, even with aggressive antithrombotic regimen. The aim of this study is to perform an analysis of the safety and effectiveness of primary angioplasty using the radial access and a high bolus dose of tirofiban. Method: From January 2006 to April 2007, 73 patients were submitted to primary angioplasty in our service using the radial access. The in-hospital and late major adverse cardiovascular events were retrospectively evaluated particularly in respect to the occurrence of hemorrhagic and vascular complications. Results: The use of the radial access was $100 \%$ successfully. Angiographic success was obtained in $95.8 \%$ of the patients. The right coronary was treated in $50 \%$ of the cases. A high bolus dose of tirofiban was used in $75 \%$ of the patients. There were 3 in-hospital cardiac deaths, but no cause of major bleeding. Local hematomas occurred in $5.4 \%$ of the cases. In the late follow-up (average of 7 months), the mortality rate was $5.4 \%$ with no complications related to the arterial puncture site or major bleeding. Conclusion: The systematic use of the transradial approach for primary angioplasty is highly efficient and safe despite the use of an aggressive anticoagulation and antithrombotic regimen including a high bolus dose of tirofiban.

DESCRIPTORS: Angioplasty, transluminal, percutaneous coronary. Radial artery. Tyrosine, analogs \& derivatives.

A angioplastia primária é a estratégia de reperfusão de escolha nos pacientes com infarto agudo do miocárdio com supradesnivelamento do segmento ST $(\text { IAM })^{1-3}$.

A intervenção coronariana é realizada tipicamente pelo acesso transfemoral. Complicações hemorrágicas e vasculares no sítio de punção femoral podem ensejar um significante aumento de complicações, duração da 
hospitalização e custo do procedimento, principalmente quando a angioplastia é realizada sob agressivo regime de anticoagulação e terapia antiplaquetária, em particular com o uso dos inibidores da glicoproteína IIb/IIla (IGP) ${ }^{4}$.

A utilização do acesso transradial fundamenta-se na redução de complicações hemorrágicas no sítio de acesso vascular e do repouso prolongado no leito. Vários estudos têm confirmado sua aplicabilidade e potenciais vantagens sobre a via femoral ${ }^{5-9}$, com equivalência de sucesso e diminuição de complicações hemorrágicas, mesmo com a utilização de um regime antitrombótico agressivo, inclusive os IGP4

Apesar de alguns resultados conflitantes, estudos têm estabelecido a segurança e a eficácia dos IGP durante a angioplastia primária ${ }^{10-13}$. As diretrizes atuais recomendam, especificamente, a utilização de abciximab durante a ICP primária como indicação classe I³.

Em pacientes com síndromes coronarianas agudas, o estudo TARGET ${ }^{14}$ demonstrou menor eficácia do tirofiban quando comparado ao abciximab. Acredita-se que isto seja devido, principalmente, à inadequada inibição plaquetária nas primeiras horas após a administração do tirofiban durante o procedimento ${ }^{14-19}$. A utilização de bolus com dose aumentada de tirofiban tem mostrado inibição plaquetária e resultados clínicos semelhantes àqueles encontrados com o abciximab ${ }^{20-22}$. A substituição do abciximab pelo tirofiban, administrado com um regime de bolus com dose aumentada, é uma estratégia promissora que pode se mostrar custo-efetiva ${ }^{21}$.

O objetivo desse estudo é avaliar a segurança e a efetividade da angioplastia primária via radial associada à utilização de bolus com dose aumentada de tirofiban.

\section{MÉTODO}

Entre janeiro de 2006 e maio de 2007, 79 pacientes com infarto do miocárdio (IAM) com supradesnivelamento do segmento ST foram submetidos à angioplastia primária na Santa Casa de Marília. Setenta e três $(92 \%)$ pacientes compuseram a amostra. Seis pacientes foram excluídos da amostra devido ao não emprego da técnica radial: três por ausência de pulso radial (dois por uso prévio da técnica de Sones e um por cateterismo e angioplastia prévios via radial direita), dois por choque cardiogênico e bloqueio atrioventricular total com necessidade de marca-passo temporário e outro que evoluiu com embolização coronariana durante um estudo hemodinâmico por valvopatia mitral.

O seguimento tardio foi obtido em todos os pacientes, por meio dos prontuários e/ou pelo contato telefônico.

A via de escolha foi a radial direita. A escolha da técnica radial ocorreu mediante a realização do teste de Allen modificado. Se negativo o teste, a outra via arterial utilizada foi a radial esquerda. Foram empregados introdutores curtos $(8 \mathrm{~cm}) 6$ French. Solução com 5000 UI de sulfato de heparina e $10 \mathrm{mg}$ de mononitrato de isossorbida foi injetada no introdutor. Os procedimentos foram realizados com as técnicas habituais. Stents foram implantados com a técnica direta ou convencional. A remoção dos introdutores ocorreu logo após os procedimentos, a hemostasia foi feita com curativo compressivo com uma bandagem elástica adesiva porosa (Tensoplast $\left.{ }^{\circledR}\right)$, por cerca de quatro horas. Após este período, a compressão foi relaxada e colocado um pequeno curativo, durante 24 horas; não houve necessidade de dispositivos hemostáticos. A circulação funcional da mão foi verificada uma hora após.

Todos os pacientes receberam aspirina $300 \mathrm{mg}$ na sala de emergência. Clopidogrel foi administrado na sala de hemodinâmica, utilizado na dose de 300 mg ou $600 \mathrm{mg}$, respectivamente, com e sem tirofiban. Heparina não fracionada foi utilizada em todos os pacientes, na dose total de $100 \mathrm{U} / \mathrm{kg}$. Tirofiban foi utilizado a critério do operador, na dose de $25 \mu / \mathrm{kg}$ em bolus de três minutos e na dose de manutenção de $0,15 \mu / \mathrm{kg} / \mathrm{min}$ por $12-18$ horas. Cateter para aspiração de trombo coronariano (Pronto ${ }^{\circledR}$ ) foi também utilizado a critério do operador.

Os desfechos utilizados foram: sucesso angiográfico (porcentual do diâmetro da estenose do vaso-alvo <20\% e fluxo coronariano no vaso-alvo TIMI 3), mortalidade hospitalar geral, complicações no acesso arterial, como sangramentos maiores (determinantes de hospitalização prolongada, intervenção cirúrgica, transfusão sanguínea ou acidente vascular cerebral hemorrágico), fístulas artériovenosas, isquemia do membro com necessidade de cirurgia vascular e falência do procedimento, definida como a necessidade de um segundo acesso arterial ou falha no procedimento por impossibilidade de canulação da artéria radial ou conclusão da angioplastia coronária ou devido a uma complicação maior no local da punção arterial.

As variáveis contínuas foram expressas em médias e desvio-padrão e, as categóricas, em números absolutos e seu porcentual.

\section{RESULTADOS}

A maioria $(73,8 \%)$ dos pacientes era do sexo masculino. Houve baixa incidência de pacientes com história prévia de infarto do miocárdio, angioplastia coronariana e cirurgia de revascularização miocárdica. Os dados demográficos estão na Tabela 1. A maioria dos pacientes era uniarterial e houve maior acometimento da artéria coronária direita (Tabela 2). Trombo angiograficamente visível ocorreu em 18 (24,6\%) pacientes, foi realizada trombectomia em apenas $22 \%$ dessa população, a partir de outubro de 2006. O regime de bolus com dose aumentada de tirofiban foi utilizado em $74 \%$ dos pacientes. Em nossa amostra, sete $(9,5 \%)$ pacientes tinham 75 anos ou mais e, destes, quatro $(5,4 \%)$ utilizaram tirofiban sem episódios de sangramento maior ou menor. Heparina não foi utilizada após o procedimen- 
to. Stents foram implantados em 69 (94,5\%) pacientes. Todos os procedimentos foram realizados com sucesso pela via radial. Os cateteres utilizados para artéria coronária esquerda e coronária direita foram os extra backup $(85 \%)$ e Judkins de direita $(86 \%)$, respectivamente. O sucesso angiográfico ocorreu em $70(95,8 \%)$ pacientes.

A Tabela 3 mostra a ocorrência de eventos adversos maiores. Houve três óbitos hospitalares. Um ocorreu 12 horas após a angioplastia realizada com suces-

TABELA 1

Características basais

\begin{tabular}{lr}
\hline Características Basais & Total $(\mathbf{n}=\mathbf{7 3})$ \\
\hline Idade (média) & $60,8 \pm 10,4$ \\
Sexo masculino (\%) & $51(69,8)$ \\
HAS (\%) & $50(68,4)$ \\
Diabetes (\%) & $15(20,5)$ \\
Dislipidemia (\%) & $26(35,6)$ \\
História familiar de ICO (\%) & $8(10,9)$ \\
IAM prévio (\%) & $4(5,4)$ \\
Tabagismo (\%) & $35(47,9)$ \\
Cirurgia de revascularização (\%) & $0(0)$ \\
Angioplastia prévia (\%) & $1(1,3)$ \\
\hline
\end{tabular}

TABELA 2

Características angiográficas e do procedimento total

\begin{tabular}{|c|c|}
\hline $\begin{array}{l}\text { Características angiográficas } \\
\text { e do procedimento }\end{array}$ & Total $(n=73)$ \\
\hline \multicolumn{2}{|l|}{ Número de vasos tratados } \\
\hline Uniarteriais (\%) & $35(47,9)$ \\
\hline Biarteriais (\%) & $23(31,5)$ \\
\hline Triarteriais (\%) & $15(20,5)$ \\
\hline \multicolumn{2}{|l|}{ Vasos tratados } \\
\hline $\mathrm{DA}(\%)$ & $30(41,1)$ \\
\hline $\mathrm{CX}(\%)$ & $6(8,2)$ \\
\hline $\mathrm{CD}(\%)$ & $37(50,6)$ \\
\hline Trombo angiográfico (\%) & $18(24,6)$ \\
\hline Tempo porta-balão (min) & $84,3 \pm 26,5$ \\
\hline \multicolumn{2}{|l|}{ Fluxo TIMI Pré } \\
\hline TIMI 0/1 pré (\%) & $61(83,5)$ \\
\hline TIMI 2/3 pré (\%) & $12(16,4)$ \\
\hline Bolus de Tirofiban $25 \mu / \mathrm{kg}(\%)$ & $54(74)$ \\
\hline Cateter de aspiração (\%) & $4(5,4)$ \\
\hline Implante de stent (\%) & $69(94,5)$ \\
\hline \multicolumn{2}{|l|}{ Fluxo TIMI Pós } \\
\hline TIMI 0/1 pós (\%) & $3(4,1)$ \\
\hline TIMI 2/3 pós (\%) & $70(95,8)$ \\
\hline Sucesso angiográfico (\%) & $70(95,8)$ \\
\hline
\end{tabular}

so angiográfico, por fibrilação ventricular refratária. O segundo óbito ocorreu, no quarto dia de internação, após insucesso angiográfico (no-reflow), por choque cardiogênico refratário. O último paciente, admitido por IAM anterior extenso com 10 horas de evolução, apresentou trombo angiográfico com mais de $15 \mathrm{~mm}$ de extensão, sem resolução por trombectomia, IGP e angioplastia coronariana, com no-reflow, foi a óbito, no segundo dia, por choque cardiogênico.

O seguimento tardio foi obtido em $70 \%$ dos pacientes. Durante o seguimento médio de sete meses, 4 (5,4\%) óbitos ocorreram. Destes, 3 ocorreram no terceiro mês após o episódio agudo e por provável etiologia cardíaca; um paciente apresentou abdome agudo inflamatório e faleceu no quarto mês após o IAM. Não houve outras complicações. Houve um episódio de trombose subaguda de stent, na primeira semana após o procedimento, tratada com nova angioplastia coronariana com sucesso clínico (Tabela 3).

Nenhum sangramento maior foi identificado no período de internação hospitalar. Não houve necessidade de intervenção cirúrgica no sítio de punção radial, transfusão sanguínea, prolongamento da internação hospitalar, acidente vascular cerebral hemorrágico ou sangramento intestinal. Ocorreram quatro hematomas no local da punção, porém sem edema, isquemia da mão. Evidenciou-se um caso de flebite localizada e responsiva a tratamento clínico.

\section{DISCUSSÃo}

O resultado dessa análise demonstra a segurança e eficácia do acesso transradial na angioplastia primária, mesmo com a utilização de tirofiban em regime de bolus com dose aumentada.

O sucesso da abordagem transradial nessa amostra decorre de sua utilização sistemática em nosso serviço. No mesmo período da análise, realizamos 327 angioplastias eletivas (Figura 1). A via radial foi utilizada em $302(92,4 \%)$ pacientes, e a via femoral nos demais. O sucesso nas angioplastias eletivas foi de $98,5 \%$, com utilização de angioplastia com balão em somente 5

TABELA 3

Ocorrência de eventos

\begin{tabular}{lc}
\hline Eventos clínicos & Total $(\mathbf{n}=\mathbf{7 3})$ \\
\hline Óbito hospitalar (\%) & $3(4,1)$ \\
Seguimento tardio (\%) & $51(69,8)$ \\
Tempo de seguimento (meses) & $7,26 \pm 4,9$ \\
Óbito tardio (\%) & $4(5,4)$ \\
Reinfarto (\%) & $1(1,3)$ \\
Revascularização de urgência (\%) & $1(1,3)$ \\
Sangramento maior & 0 \\
Sangramento menor & $4(5,4)$ \\
\hline
\end{tabular}




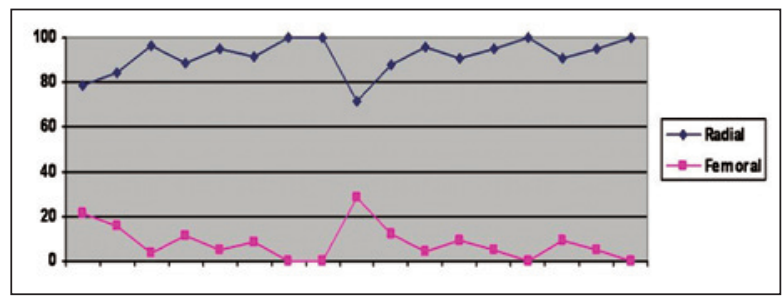

Figura 1 - Vias de acesso utilizadas nas angioplastias eletivas, no período de janeiro de 2006 a maio de 2007.

$(1,5 \%)$ pacientes. Os introdutores de calibre $6 \mathrm{~F}$ foram utilizados em 294 (97,3\%) pacientes, em 3 (0,9\%) doentes utilizamos introdutores $5 \mathrm{~F}$ e, em 5 (1,5\%), introdutores de calibre 7F. Quando não foi possível a utilização da via radial direita, optamos pela artéria radial esquerda para a realização do procedimento. Em casos de necessidade de marca-passo temporário, utilizamos o acesso radial associado à punção da veia jugular ou mesmo da punção da veia femoral.

A utilização rotineira dessa via de acesso para a realização de angioplastia primária somente foi implantada após um período de curva de aprendizagem dos operadores, em acordo com vários estudos que evidenciam sua importância ${ }^{23-26}$.

Em nosso grupo de pacientes, houve predomínio de IAM de parede inferior, em sua maioria estáveis clinicamente. Uma eventual maior instabilidade clínica potencialmente contribuiria negativamente com o sucesso clínico e determinaria maior necessidade de balão intra-aórtico, que poderia resultar em menor utilização do acesso radial.

Embora se constitua uma opção há mais de dez anos para a realização de angioplastia coronariana, em nosso meio, a via radial continua a ser pouco utilizada. Araújo e Mangione ${ }^{27}$ avaliaram 53.777 pacientes submetidos à intervenção coronariana cadastrados na CENIC (Centro Nacional de Intervenções Cardiovasculares). Destes, 48.455 pacientes foram submetidos à angioplastia coronariana eletiva e 5.322, na vigência de infarto. A via radial foi utilizada em apenas $10,1 \%$ dos casos eletivos e $4,6 \%$ nos pacientes com infarto, sendo a via femoral utilizada em $87,7 \%$ dos casos eletivos e em $91,7 \%$ dos casos de urgência. Notou-se que, em nosso país, a via radial é utilizada em pacientes selecionados (homens, com lesões únicas e sem revascularização cirúrgica prévia). Pacientes com doenças mais graves são eleitos para o acesso femoral.

Após sua introdução por Kiemeneij and Laarman ${ }^{28}$, o benefício da técnica radial tem sido claramente demonstrado em diversos estudos ${ }^{7}$. As complicações hemorrágicas no sítio de acesso são virtualmente eliminadas. Após o procedimento, a deambulação e a alta hospitalar se fazem mais precoces ${ }^{8}$. A morbidade do procedimento é menor e os pacientes de forma preponderante preferem-na à abordagem transfemoral ${ }^{8,29}$.
Vários estudos demonstram a eficácia e a segurança da via radial em pacientes submetidos à angioplastia primária ${ }^{4,30,31}$. Saito et al. ${ }^{31}$ randomizaram 213 pacientes com IAM dentro de 12 horas do início dos sintomas: 77 tratados por via radial (ITR) e 72 tratados por via femoral (ITF). A taxa de sucesso da reperfusão e a incidência de eventos cardíacos maiores (morte, IAM não fatal, repetida revascularização), durante a hospitalização, foram similares $(96,1 \%$ e $5,2 \%$ vs. $97,1 \%$ e $8,3 \%)$, nos grupos ITR e ITF, respectivamente $(p=0,624$ e $p=0,444)$.

Philippe et al. ${ }^{4}$ compararam também os resultados clínicos da intervenção transradial (ITR) e transfemoral (ITF), na angioplastia primária com uso de abciximab. Foram, prospectivamente, avaliados 119 pacientes (ITR $\mathrm{n}=64$ e ITF $\mathrm{n}=55$ ). Nesse estudo não randomizado, a sobrevida livre de eventos foi semelhante para ambas as técnicas (97\% vs. 94,5\%; p=0,19). Não houve complicações hemorrágicas maiores no grupo da ITR, o oposto do que foi encontrado no grupo da $\operatorname{ITF}(5,5 \%, p=0,03)$. O tempo de hospitalização também foi significantemente maior no grupo da ITF $(5,9 \pm 2,1$ vs. 4,5 $\pm 1,2$ dias; $p=0,05)$.

Os inibidores da glicoproteína Ilb/IIla são uma terapêutica adjunta de extrema importância no tratamento do IAM, embora resultados conflitantes não demonstrem benefícios de redução na mortalidade, quando comparados ao implante de stent ${ }^{12}$. O abciximab tem sido o fármaco indicado como classe I, mas devido ao seu alto custo é pouco utilizado. Na comparação com abciximab, o tirofiban nas doses habituais (bolus de $10 \mu \mathrm{g} / \mathrm{kg}$ e infusão de $0,15 \mu \mathrm{g} / \mathrm{kg} / \mathrm{min}$ ) foi menos efetivo, principalmente em pacientes com síndrome coronária aguda ${ }^{14}$. A menor incidência de eventos proporcionada pelo abciximab nesse estudo é devida à menor incidência de IAM periprocedimento. Diferenças foram aparentes dentro de 12 horas até, no máximo, por 24 horas depois do procedimento. Foi encontrado que a extensão da inibição da agregação foi maior de 15 até 60 minutos do início do tratamento com abciximab. Schneider et al. ${ }^{16}$ avaliaram a dose do bolus do tirofiban capaz de produzir uma inibição da agregação maior do que $90 \%$, durante a primeira hora do tratamento, em pacientes com síndrome coronária aguda que fizeram ICP. Foram testadas duas dosagens; bolus de $20 \mu \mathrm{g} / \mathrm{kg}$ e infusão de $0,15 \mu \mathrm{g} / \mathrm{kg} / \mathrm{min}$ por $18-24$ horas ou bolus de $25 \mu \mathrm{g} / \mathrm{kg}$ com a mesma infusão. A segunda dosagem demonstrou uma inibição da agregação maior de $90 \%$ na primeira hora. Com o regime utilizado no estudo TARGET, a extensão média (bolus de $10 \mu \mathrm{g} / \mathrm{kg}$ e infusão de $0,15 \mu \mathrm{g} / \mathrm{kg} / \mathrm{min}$ ) da inibição da agregação em resposta a $20 \mu \mathrm{M}$ de ADP foi 90-94\%, no período de 15 a 60 minutos após o início do tratamento com abciximab, e, 60-66\%, depois do tirofiban $^{17,18}$. O intervalo de 15-60 minutos depois do início do tratamento é um período crítico, durante o qual ocorre lesão arterial provocada pelo balão e um corpo estranho metálico, potencialmente trombogênico 
(stent), é freqüentemente implantado. Dessa maneira, a inibição máxima da agregação plaquetária, neste período, traria um benefício adicional.

Srinivas et al. ${ }^{32}$ avaliaram o registro de 7.321 pacientes que fizeram ICP primária no estado de Nova Iorque; $78,5 \%$ dos quais utilizaram IGP. A mortalidade hospitalar foi significativamente menor nos pacientes que utilizaram IGP ( $3 \%$ vs. $6,2 \%, p<0,0001)$. Em 2.837 pacientes, foi avaliado o tipo de agente utilizado nas angioplastias primárias: $33 \%$ receberam abciximab, $46 \%$, eptifibatide ou tirofiban e $21 \%$, não receberam nenhuma medicação. A taxa de mortalidade foi menor naqueles que utilizaram abciximab e eptifibatide ou tirofiban comparada com pacientes que não receberam IGP (3,1\% vs. 2,4\% vs. 4,7\%, respectivamente, $\mathrm{p}=0,03)$.

São limitações do estudo seu caráter retrospectivo e a parcialidade do seguimento clínico tardio da população. O número de pacientes idosos, teoricamente mais propensos a complicações com a utilização dos IGP, foi pequeno. Devido à utilização sistemática da via radial em nosso serviço, não foi possível sua comparação com a via femoral.

\section{CONCLUSÃO}

No nosso serviço de cardiologia intervencionista, que utiliza a via radial sistematicamente, seu emprego na angioplastia primária é altamente eficaz e seguro, a despeito do uso de um regime de anticoagulação e antiagregação agressivos.

\section{REFERÊNCIAS BIBLIOGRÁFICAS}

1. Keeley EC, Boura JA, Grines CL. Primary angioplasty versus intravenous thrombolytic therapy for acute myocardial infarction: a quantitative review of 23 randomised trials. Lancet. 2003;361(9351):13-20.

2. Sociedade Brasileira da Cardiologia. III Diretriz sobre tratamento do infarto agudo do miocárdio. Arq Bras Cardiol. 2004;83 (Suppl IV):S1-86.

3. Antman EM, Anbe DT, Armstrong PW, Bates ER, Green LA, Hand $M$, et al. ACC/AHA guidelines for the management of patients with ST-elevation myocardial infarction: a report of the American College of Cardiology/American Heart Association Task Force on Practice Guidelines (Committee to Revise the 1999 Guidelines for the Management of Patients with Acute Myocardial Infarction). Circulation. 2004;110(9):e82-292.

4. Philippe F, Larrazet F, Meziane T, Dibie A. Comparison of transradial $v s$. transfemoral approach in the treatment of acute myocardial infarction with primary angioplasty and abciximab. Catheter Cardiovasc Interv. 2004;61(1):67-73.

5. Lotan C, Hasin Y, Mosseri M, Rozenman Y, Admon D, Nassar $\mathrm{H}$, et al. Transradial approach for coronary angiography and angioplasty. Am J Cardiol. 1995;76(3):164-7.

6. Barbeau GR, Carrier G, Ferland S, Letourneau L, Gleeton O, Lariviere MM. Right transradial approach for coronary procedures: preliminary results. J Invasive Cardiol. 1996;8(suppl): 19D-21D.

7. Kiemeneij F, Laarman GJ. Transradial artery Palmaz-Schatz coronary stent implantation: results of a single-center feasibility study. Am Heart J. 1995;130(1):14-21.
8. Kiemeneij F, Laarman GJ, Odekerken D, Slagboom T, van der Wicken R. A randomized comparison of percutaneous transluminal coronary angioplasty by the radial, brachial and femoral approaches: the access study. J Am Coll Cardiol. 1997;29(6):1269-75.

9. Mann T, Cubeddu G, Bowen J, Schneider JE, Arrowood M, Newman WN, et al. Stenting in acute coronary syndromes: a comparison of radial versus femoral access sites. J Am Coll Cardiol. 1998;32(3):572-6.

10. De Luca G, Suryapranata H, Stone GW, Antoniucci D, Tcheng JE, Neumann FJ, et al. Abciximab as adjunctive therapy to reperfusion in acute ST-segment elevation myocardial infarction: a meta-analysis of randomized trials. JAMA. 2005;293(14):1759-65

11. Montalescot G, Borentain M, Payot L, Collet JP, Thomas D. Early vs. late administration of glycoprotein Ilb/IIla inhibitors in primary percutaneous coronary intervention of acute STsegment elevation myocardial infarction: a meta-analysis. JAMA. 2004;292(3):362-6.

12. Stone GW, Grines CL, Cox DA, Garcia E, Tcheng JE, Griffin $\mathrm{J}$, et al. Comparison of angioplasty with stenting, with or without abciximab, in acute myocardial infarction. $N$ Engl J Med. 2002;346(13):957-66.

13. Kandzari DE, Hasselblad V, Tcheng JE, Stone GW, Califf RM, Kastrati A, et al. Improved clinical outcomes with abciximab therapy in acute myocardial infarction: a systematic overview of randomized clinical trials. Am Heart J. 2004;147(3):457-62.

14. Topol EJ, Moliterno DJ, Herrmann HC, Powers ER, Grines $\mathrm{CL}$, Cohen DJ, et al. Comparison of two platelet glycoprotein Ilb/IIla inhibitors, tirofiban and abciximab, for the prevention of ischemic events with percutaneous coronary revascularization. Investigadores do estudo TARGET. N Engl J Med. 2001;334(25):1888-94.

15. Lakkis N, Lakiss N, Bobek J, Farmer J. Platelet inhibition with tirofiban early during percutaneous coronary intervention: dosing revisited. Catheter Cardiovasc Interv. 2002;56(4): 474-7.

16. Schneider D, Herrmann $H$, Lakkis $N$, Aguirre $F$, Wan $Y$, Aggarwal A, et al. Enhanced early inhibition of platelet aggregation with an increased bolus of tirofiban. Am J Cardiol. 2002;90(12):1421-3.

17. Herrmann HC, Swierkosz TA, Kapoor S, Tardiff DC, DiBattiste PM, Hirshfeld JW, et al. Comparison of degree of platelet inhibition by abciximab versus tirofiban in patients with unstable angina pectoris and non-Q-wave myocardial infarction undergoing percutaneous coronary intervention. Am J Cardiol. 2002;89(11):1293-7.

18. Kabbani SS, Aggarwal A, Terrien EF, DiBattiste PM, Sobel BE, Schneider DJ. Suboptimal early inhibition of platelets by treatment with tirofiban and implications for coronary interventions. Am J Cardiol. 2001;89(5):647-50.

19. Schneider DJ, Herrmann HC, Lakkis N, Aguirre F, Lo MW, Yin $\mathrm{KC}$, et al. Increased concentrations of tirofiban in blood and their correlation with inhibition of platelet aggregation after greater bolus doses of tirofiban. Am J Cardiol. 2003;91(3):334-6.

20. Bolognese L, Falsini G, Liistro F, Angioli P, Ducci K, Taddei $\mathrm{T}$, et al. Randomized comparison of upstream tirofiban versus downstream high bolus dose tirofiban or abciximab on tissuelevel perfusion and troponin release in high-risk acute coronary syndromes treated with percutaneous coronary intreventions. The EVEREST trial. J Am Coll Cardiol. 2006;47 (3):522-8.

21. Valgimigli M, Percoco G, Malagutti P, Campo G, Ferrari F, Barbieri D, et al. Tirofiban and sirolimus-eluting stent vs. abciximab and bare-metal stent for acute myocardial infarction: a randomized trial. JAMA. 2005;293(17):2109-17. 
22. Danzi G, Sesana M, Capuano C, Mauri L, Berra Centurini $\mathrm{P}$, Baglini R. Comparison in patients having primary coronary angioplasty of abciximab versus tirofiban on recovery of left ventricular function. Am J Cardiol. 2004;94(1):35-9.

23. Goldberg SL, Renslo R, Sinow R, French WJ. Learning curve in the use of the radial artery as vascular access in the performance of percutaneous transluminal coronary angioplasty. Cathet Cardiovasc Diagn. 1998;44(2):147-52.

24. Louvard Y, Lefèvre T, Morice MC. Radial approach: what about the learning curve? Cathet Cardiovasc Diagn. 1997; 42(4):467-8

25. Teixeirense PB, Gubolino LA, Bragalha AMLA, Toledo JFB, Franceschine J, Colombo $\mathrm{O}$, et al. Análise temporal dos resultados imediatos com a aplicação da punção transradial na intervenção coronária percutânea. Rev Bras Cardiol Invas. 2006;14(4):380-5.

26. Nunes GL, Oliveira AT, Alves L, Alfonso T. Influência da curva de aprendizado no sucesso e na ocorrência de complicações associadas aos procedimentos pela via radial. Rev Bras Cardiol Invas. 2007;15(2):115-8.

27. Araújo CM, Mangione JA. Diferenças e semelhanças dos resultados de acordo com as diferentes vias de acesso. Rev Bras Cardiol Invas. 2006;14(3):273-9.
28. Kiemeneij F, Laarman GJ. Percutaneous transradial artery approach for coronary stent implantation. Cathet Cardiovasc Diagn. 1993;30(2):173-8.

29. Cooper CJ, El-Shiekh RA, Blaesing LD. Patient preference for cardiac catheterization via the transfemoral approach. J Am Coll Cardiol. 1997;29(Suppl A):310A.

30. Louvard Y, Ludwig J, Lefèvre T, Schmeisser A, Brück $M$, Scheinert D, et al. Transradial approach for coronary angioplasty in the setting of acute myocardial infarction: a dual-center registry. Catheter Cardiovasc Interv. 2002;55(2): 206-11.

31. Saito S, Tanaka S, Hiroe Y, Miyashita Y, Takahashi S, Tanaka K, et al. Comparative study on transradial approach vs. transfemoral approach in primary stent implantation for patients with acute myocardial infarction: results of the test for myocardial infarction by prospective unicenter randomization for access sites (TEMPURA) trial. Catheter Cardiovasc Interv. 2003;59(1):26-33.

32. Srinivas VS, Skeif B, Negassa A, Bang JY, Shaqra H, Monrad ES. Effectiveness of glycoprotein IIb/IIla inhibitor use during primary coronary angioplasty: results of propensity analysis using the New York State Percutaneous Coronary Intervention Reporting System. Am J Cardiol. 2007;99(4):482-5. 